\title{
Effect of Seaweed Fertilizer 'SUNRED' on the Fruit Quality of 'Red Globe' Grape
}

\author{
Lu Yuan 1, 3, a , Xiulan Lv',b, Jin Wang', \\ ${ }^{1}$ College of Horticulture, Sichuan Agricultural University, Chengdu, Sichuan, China \\ ${ }^{2}$ Institute of Pomology and Olericulture, Sichuan Agricultural University, Chengdu, China \\ ${ }^{3}$ Chengdu Agricultural College, Chengdu, Sichuan, China \\ a1054069372@qq.com, bxllvjj@163.com, c251040278@qq.com, dllj800924@163.com, \\ e1324856299@qq.com. *Corresponding author.
}

\begin{abstract}
Keywords: SUNRED; Red Globe; soluble solids; fruit quality
Abstract: The experimental material is 'Red Globe' grape that is taking shelter from rain. Use different concentrations of SUNRED on the grapes, when the color of grape is turning. Then study the effect of color and quality of fruit. The results show that, SUNRED 1000 times could promote the grape coloring. The total chlorophyll content of SUNRED 1000 treated peel was the lowest, $4.8 \mathrm{mg} / \mathrm{g}$, and the anthocyanin content was the highest, $1785 \mathrm{nmol} / \mathrm{g}$. It improved the color index of grape fruit .
\end{abstract}

\section{Introduction}

'Red Globe' belongs to the European and Asian varieties of table grapes, originating in California, USA. The 'Red Earth' grape is a mid-to-late ripe fresh variety with high yield, good quality, and storage and transportation.

In southern China, there is plenty of rain and high temperatures during the summer, and the duration of light is shorter than that of other regions. The temperature difference between day and night is small, and the diseases of open-field cultivated grapes are serious, resulting in low yield, poor appearance and inherent quality of the fruit, European and Asian grape varieties are more difficult to develop in regions with an average annual rainfall of over $600 \mathrm{~mm}$. Thus limiting the sustainable and healthy development of the grape industry in the southern region ${ }^{[1]}$. The introduction of cultivation model of taking shelter from rain has solved the problems caused by the large amount of rainfall, high temperature and high humidity in southern China, and the serious problems of fruit drop and fruit cracking. It also provides environmental conditions for the cultivation of European and Asian grape varieties in the southern region. With the promotion of taking shelter from rain cultivation techniques in southern China, 'Red Globe' has become the largest variety in southern viticulture. In the Sichuan Basin, which is characterized by high temperature and rain, low temperature difference between day and night, and low sunshine, the cultivation of taking shelter from rain has become the most important cultivation mode for grapes.

Compared with open cultivation, cultivation of taking shelter from rain reduces crown light intensity, soil moisture, and orchard wind speed due to the canopy cover film. It affect the photosynthesis, root growth of the leaves and thus appear the fruit coloring is light and sugar content is reduced and other issues ${ }^{[2]}$. In the past, production of abscisic acid was mostly used to improve the fruit quality of cultivating grapes. However, the blind use of plant growth regulators will not only improve the grape quality, but also cause problems such as falling flowers, falling fruit, poor coloration. In addition, studies have shown that the application of seaweed fertilizer on crops such as peanuts, corn, purple sweet potatoes and vegetables can increase production and quality ${ }^{[3-6]}$. The application and research of seaweed fertilizer on fruit trees is rarely reported.

In recent years, Pengshan District, where the main grape-producing areas of Sichuan sheltered from raining, fruit growers began to spray SUNRED, a seaweed fertilizer developed by Italy's Biolchim s.r.1. for coloring and sugar-supplementing of grapes. However, at home and abroad, SUNRED promotes coloration of seaweed fertilizer. However, there are no reports in the literature about the 
seaweed fertilizer SUNRED promoting color-reducing sugar at home and abroad. Therefore, in this experiment, 'Red Globe' in Guoyuan Village, Guanyin Town, Pengshan District of Sichuan Province was used as a test material. In the start of fruit coloring, 'Red Globe' grapes were treated with different concentrations of SUNRED to investigate its fruit quality on 'Red Globe' grapes. By increasing the value of goods, social and economic benefits are increased. Reliable basis for the application and promotion of seaweed fertilizer SUNRED in high quality and efficient safety production of southern grapes are provided.

\section{Materials and Methods}

Materials and sampling. The experimental park is located in the Grape Demonstration Park in Pengshan County, Meishan City, of Sichuan Province. It is 600 meters above sea level, subtropical humid climate, mild climate, abundant rainfall, and four distinct seasons. The experimental trees are "Red Globe" grape (medium and late ripening variety) cultivated for 7 years, that are taking shelter from rain. The planting spacing is $2 \mathrm{~m} \times 3 \mathrm{~m}$, and the tree shaped is $\mathrm{Y}$. And the experimental trees have strong tree vigor and the general cultivation and management are basically the same. At the beginning of coloring, select representative and uniform-sized clusters to hung up the tag, and sprayed SUNRED firstly. The different treat were water, SUNRED 600 times, SUNRED 800 times, SUNRED 1000 times. After 10 days, sprayed SUNRED secondly using the same spraying method at the first time. And before spraying at that day, began to sample. One sample every five days until fruit ripening. Each time, picked 20 even-sized grapes from Top, middle, and bottom of the tree. At young fruit stage, picked 120 grapes. After fruit expanding, picked 90 grapes. Brong samples back to the lab with an ice box and peeled the peel and flesh for storage in a refrigerator at $-80^{\circ} \mathrm{C}$.

Determination of single fruit weight and vertical and horizontal diameter of fruit. At the fruit maturity, 20 grapes which were normal-developed, disease-free pests were harvested and weighed on an electronic balance $(0.1 \mathrm{~g})$ to record the mass of each fruit $(\mathrm{g})$. Finally, the average fruit weight of each fruit at different developmental stages was measured. Then use the vernier caliper to measure the vertical and horizontal diameters of the fruit.

Determination of Vc content. Vc was measured using dichlorophenol indophenol sodium titration method $^{[7]}$.

Determination of titratable acid. Use Standard acid-base titration method, which references Xiong Qinge $^{[8]}$.

Soluble solids (TSS). The fruit TSS was measured using a refractometer method ${ }^{[7]}$, and repeated three times.

\section{Results and Discussion}

Effects of different concentrations of SUNRED on the fruit size of 'Red Globe' grape during fruit maturity. As shown in Figure 1, at the fruit maturity of 'Red Globe' (August 19), the single fruit weight treated with SUNRED 1000 times and the vertical and horizontal diameters of the fruits were the largest, which wre $16.3 \mathrm{~g}, 32.18 \mathrm{~mm}$, and $27.31 \mathrm{~mm}$, respectively. The difference between treatments was significant. And the single fruit weight and the vertical and horizontal diameters of fruits treated with SUNRED 600 times were the smallest, which were $11 \mathrm{~g}, 29.02 \mathrm{~mm}$, and $24.25 \mathrm{~mm}$, respectively, which were significantly different from other treatments. It shows that the SUNRED 1000 times could significantly increase the single fruit weight and vertical and horizontal diameters of the 'Red Globe' grape fruit, while the SUNRED 600 times was reduced due to the high concentration. 


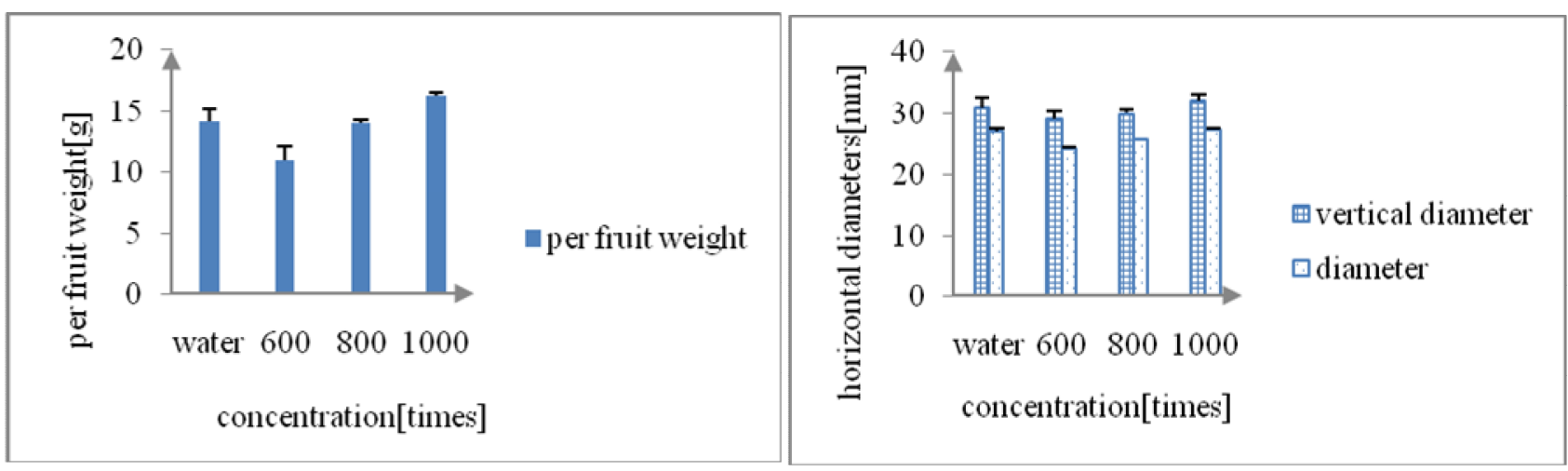

Figure 1. Different concentrations SUNRED on the 'Red Globe' grape fruit's size

Effects of different concentrations of $S U N R E D$ on Vc content of 'Red Globe' grape fruit. As can be seen from Figure 2, as the fruit matures, the Vc content increases first and then decreases, tending to be stable at the fruit maturity. The Vc content increased from July 15 to July 25, and reached the highest on July 25, and was significantly higher than that of water at SUNRED 1000 times and SUNRED 800 times, respectively $0.054 \mathrm{mg} / 100 \mathrm{~g}$ and $0.056 \mathrm{mg} / 100 \mathrm{~g}$. Afterwards, it declined, and it remained stable during the fruit maturity. The difference between treatments was not significant. This shows that spraying of SUNRED 800 times and SUNRED 1000 times could increase the content of Vc.

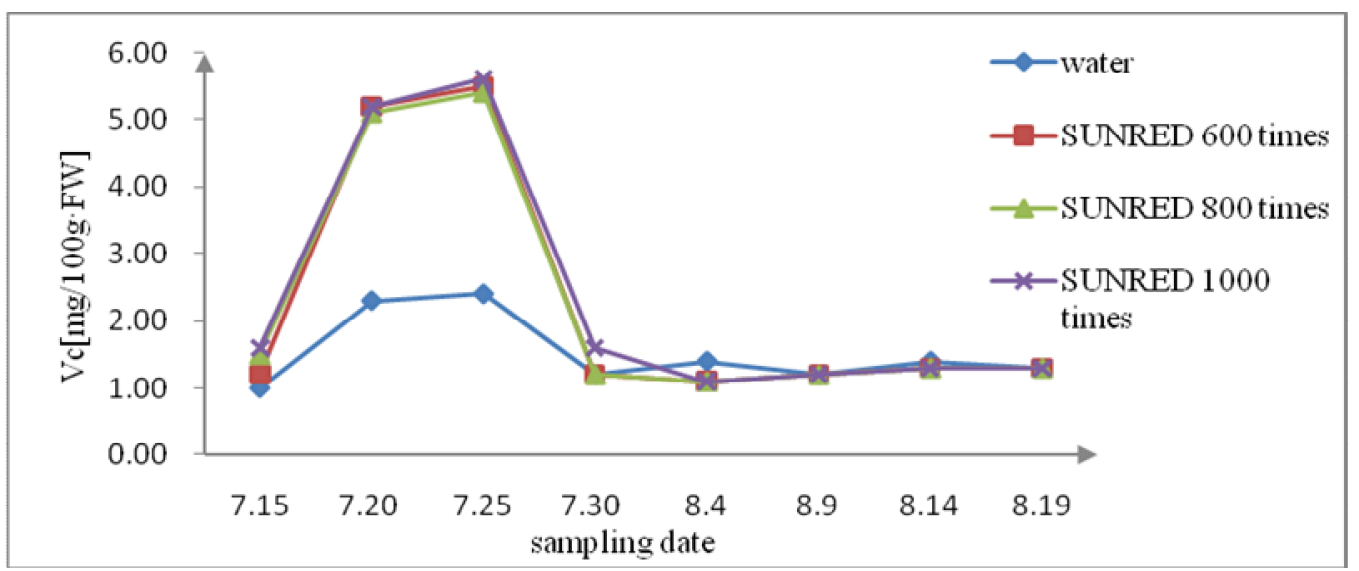

Figure 2. Effects of different concentrations SUNRED on the Vc of 'Red Globe' grape

Effects of different concentrations of $S U N R E D$ on total acid content of 'Red Globe' grape fruit. After treatment, as the fruit matures, the titratable acid content gradually decreased and was minimized during the fruit maturity. The total acid content of grape fruits treated at SUNRED 1000 times in each period was significantly lower than that of water. As shown in Figure 3, at the fruit maturity on August 19, the total acid contents at SUNRED 600 times, SUNRED 800 times, and SUNRED 1000 times treatments were significantly lower than those of water, which were $0.3659 \%$, $0.3326 \%$, and $0.3326 \%$, respectively. This shows that the SUNRED treatment of fruits could significantly reduce the total acid content of fruits, and SUNRED 1000 times the effect was particularly obvious. 


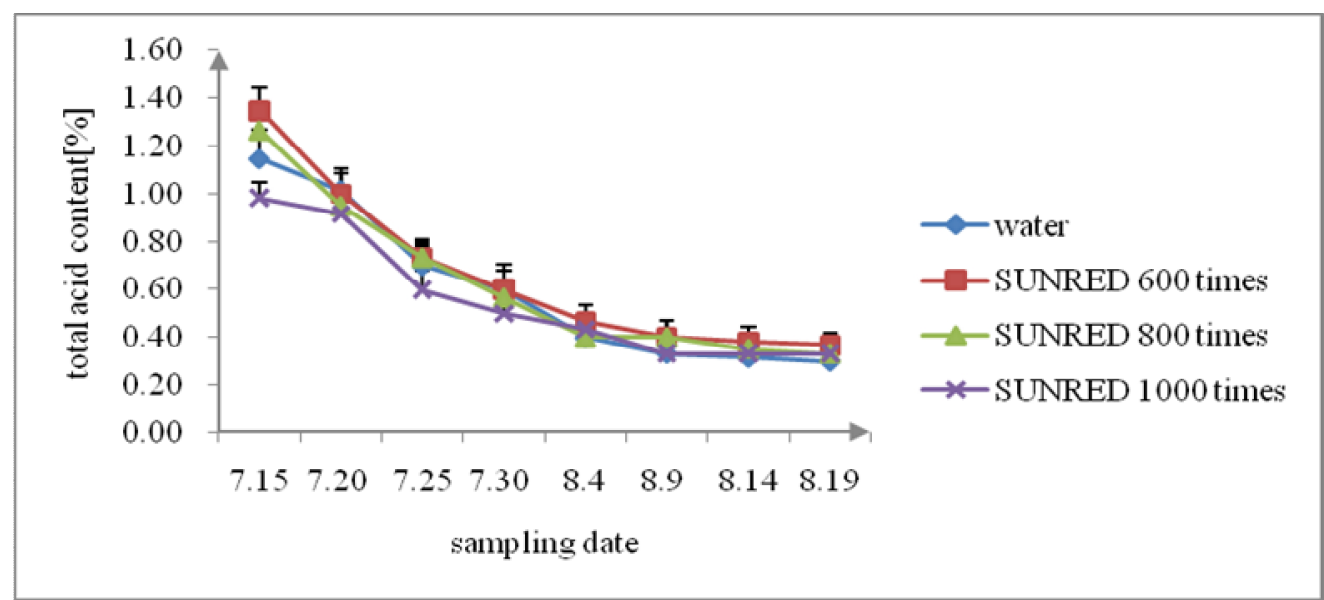

Figure 3. Effects of different concentrations SUNRED on the total acid of 'Red Globe' grape Effects of different concentrations of SUNRED on soluble solids of 'Red Globe' grape fruit. Soluble solids are one of the important indicators for judging fresh grapes. From figure 4, it can be seen that the content of soluble solids gradually increased with fruit growth and tends to be stable at the fruit maturity. The soluble solids content of fruits of SUNRED 1000 times treatment in each period was significantly higher than that of the water and the other treatments. At the maturity of August 19, the treatment at SUNRED 600 times and SUNRED 1000 times were significantly higher than water, which was $15.16 \%$ and $15.61 \%$, respectively. This shows that the SUNRED treatment could increase the soluble solid content of grapes, and the SUNRED 1000 times was the most obvious effect.

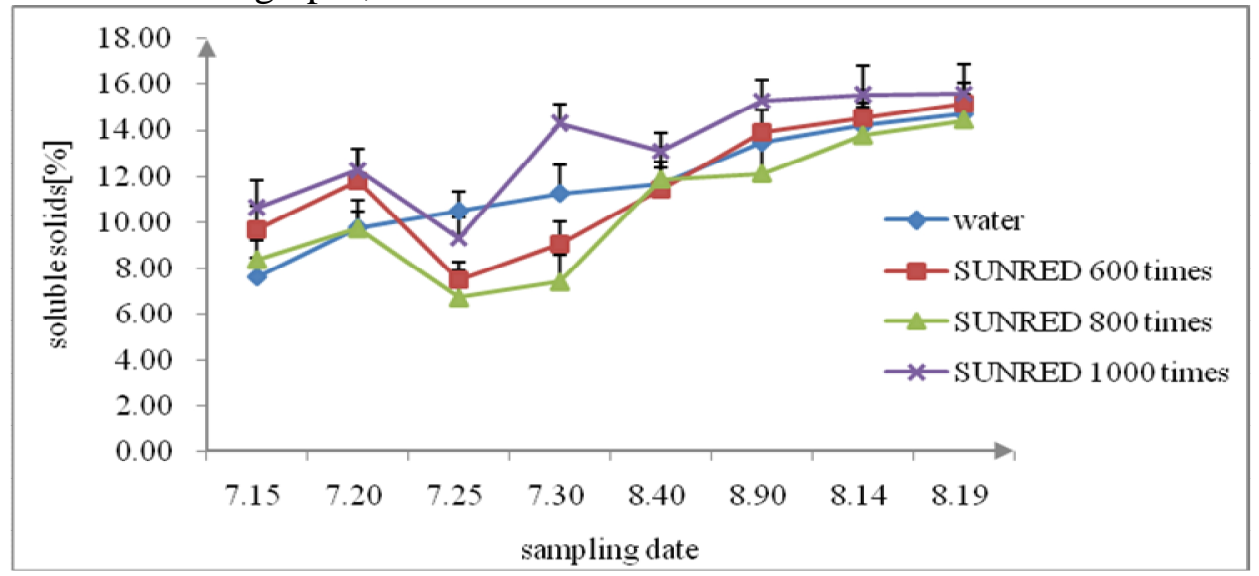

Figure 4. Effects of different concentrations SUNRED on the TSS of 'Red Globe' grape

\section{Conclusions}

Fruit vertical and horizontal diameter, single fruit weight, soluble solids, total acid, Vc, and solid-acid ratio are important indicators of fruit quality. At the same time, carbohydrates regulate the synthesis of anthocyanins. In this experiment, seaweed fertilizer SUNRED is used to improve the fruit shape, $\mathrm{Vc}$ and soluble solids content of the 'Red Globe' grapes that are sheltered from rain and reduce the total acid content of the fruits, thereby improving fruit quality.

By comprehensively comparing the intrinsic qualities of fruits, the spraying of SUNRED 1000 times at the start of 'Red Globe' grape coloration has the best effect on fruit quality, and it can be applied to improve the fruit quality of grapes, which are taking shelter from rain.

Under conditions of sheltering in the southwest, we can choose SUNRED which is not toxic to the environment and human body to promote the coloration and sugar increase of grapes. At the same time, in combination with traditional cultivation techniques, leaves of functional leaves are preserved to increase the accumulation of photosynthetic products. And in the period of fruit coloration, we should do a good job of hydrophobic work in the rainy season to reduce the groundwater level and ensure the normal growth of plants, thereby promoting the coloration and improving the commercial quality of the fruit. 


\section{Acknowledgements}

This work was financially supported by Sichuan Science and Technology Infrastructure Project "Research on the Digital Descriptive Standard of Grape Resources and the Construction of Sharing Platform" (Item No. 18PTDJ0140) and Sichuan Provincial Agricultural Science and Technology Achievements Transformation Fund Project "Xiahei's Promotion and Standardization Demonstration Base for New Grape Varieties"

\section{References}

[1] Fuchen Yang: Coloring Mechanism and Regulation of Grape Seed of Eurasian Grape in South China.(thesis of master digree: Anhui Agricultural University, Hefei China 2007)

[2] Jinchao Li, Luoshun Duan, Xiaoshen Zhang: The effect of sheltering rain on grape diseases and light intensity. Chinese Journal of Fruit Science. CSCD. Forum Vol. 6(2009), p847-850

[3] Gang Liu, Guiming Hou, Jun Liu: Seaweed fertilizer on the greenhouse yield and quality of melon impact. Shandong Agricultural Sciences. Forum Vol. 10(2014), p81-82

[4] Hongjie Tang, Yuheng Xu, Ximin Yao: Effects of different concentrations of seaweed fertilizer on the yield and quality of purple sweet potato. Bulletin of Agricultural Science and Technology. Forum Vol. 11(2014), p49.51

[5] Jialei Zhang, Xiangdong Li, Chuanting Yang: Effect of Paclobutrazol and Seaweed Fertilizer on Yield and Quality of Different Quality Peanuts

Influence. Chinese Journal of Oil Crop Sciences. CSCD. Forum Vol. 3(2015), p322-328

[6] Qingmei Huang, Zhiwen Xiao, Junjiao Guan: Seaweed fertilizer on corn yield and agronomic traits. Southwest Agriculture Journal. CSCD. Forum Vol. 3(2015), p1166.1170

[7] Yun Cheng, Xinxin Wu, Baijian Li: The effect of exogenous abscisic acid on the coloration and quality of Wei Ke grape. Jiangsu Agricultural Sciences. Forum Vol. 42(2014), p163-166

[8] Qinge Xiong: Plant physiology experiment tutorial ( $1^{\text {st }}$ edition, Sichuan Science and Technology Press, Chengdu, china 2003) 